\title{
Chronic Thromboembolic Pulmonary Hypertension after Pulmonary Embolism, Thrombolysis, Catheter Fragmentation, and Embolectomy
}

\author{
Christoph B. Wiedenroth ${ }^{1}$ Stefan Guth ${ }^{1} \quad$ Andreas Rolf $^{2}$ Eckhard Mayer ${ }^{1}$ \\ ${ }^{1}$ Department of Thoracic Surgery, Kerckhoff-Clinic, Bad Nauheim, \\ Hessen, Germany \\ 2 Department of Cardiology, Kerckhoff-Clinic, Bad Nauheim, Hessen, \\ Germany \\ Address for correspondence Dr. Christoph B. Wiedenroth, \\ Department of Thoracic Surgery, Kerckhoff-Clinic, Beneke Str. 2-8 \\ D-61231 Bad Nauheim, Hessen, Germany \\ (e-mail: christoph.wiedenroth@gmail.com).
}

Thorac Cardiovasc Surg Rep 2014;3:55-57.

\begin{abstract}
Keywords

- pulmonary embolism

- chronic thromboembolic pulmonary hypertension

- pulmonary endarterectomy

A 21-year-old male patient with massive acute pulmonary embolism was treated by thrombolysis, interventional thrombus fragmentation, and surgical pulmonary embolectomy. Within the following 2 years, the patient developed progressive dyspnea at exertion. Chronic thromboembolic pulmonary hypertension was diagnosed by rightheart catheter, VQ scan, magnetic resonance, and conventional pulmonary angiography. A normalization of the patient's exercise capacity and pulmonary hemodynamics could be achieved by pulmonary endarterectomy and patch reconstruction of the right main pulmonary artery.
\end{abstract}

\section{Case Description}

A 21-year-old male patient (marathon runner) with progressive dyspnea over 8 weeks was admitted to hospital on an emergency basis. The initial examination at admission revealed cardiopulmonary dysfunction with normal blood pressure, tachycardia (112 bpm) and hypoxia ( $\mathrm{po}_{2} 50.1 \mathrm{~mm} \mathrm{Hg}$ ), and an elevation of Ddimeres $(5.5 \mathrm{mg} / \mathrm{L})$. Echocardiography, chest computed tomographic scan, and a duplex sonography of the lower extremity veins showed a massive, bilateral pulmonary embolism with right ventricular dysfunction (right ventricular systolic pressure: $90 \mathrm{~mm} \mathrm{Hg}$ ) and a concomitant popliteal vein thrombosis. Screening for thrombophilia was negative.

The patient was treated systemically with heparin and $120 \mathrm{mg}$ of recombinant tissue plasminogen activator (rt-PA), that is, recombinant tissue plasminogen activator (Alteplase) intravenously followed by catheter-based thrombus fragmentation. As the patient remained in critical clinical condition, he was transferred to a cardiac surgical center and underwent emergent bilateral pulmonary embolectomy, right ventricular thrombectomy, and persistent foramen ovale (PFO) closure.
Because of tearing, the right pulmonary artery was reconstructed by a bovine pericardial patch. Postoperatively, the patient was anticoagulated orally with phenprocoumon.

Although the patient's clinical condition improved, he never reached his former physical capacity. Therefore, he consulted several pulmonologists and cardiologists for further evaluation of his dyspnea. Pulmonary function tests and blood gas analysis were normal. Echocardiography showed progressive right ventricular dysfunction, and multiple mismatched pulmonary perfusion defects were detected by VQ scan. Chronic thromboembolic pulmonary hypertension (CTEPH) was suspected 2 years after the initial event.

At admission in our clinic, the patient was in WHO functional class II. Echocardiography and magnetic resonance imaging (MRI) showed right ventricular dysfunction (tricuspid annular plane systolic excursion [TAPSE]: $13 \mathrm{~mm}$, right ventricular ejection fraction [RVEF]: $37 \%$ ). In addition, peripheral perfusion defects were detected by VQ scan. Besides, a right pulmonary artery stenosis was found by MRI ( - Fig. 1). Right heart catheterization revealed borderline pulmonary hypertension at rest, pathological increase of pulmonary received

February 8, 2014

accepted

March 18, 2014

published online

May 29, 2014
DOI http://dx.doi.org/

10.1055/s-0034-1376176. ISSN 2194-7635. (c) 2014 Georg Thieme Verlag KG
Stuttgart · New York

License terms

(1) $\circledast \circledast$ 


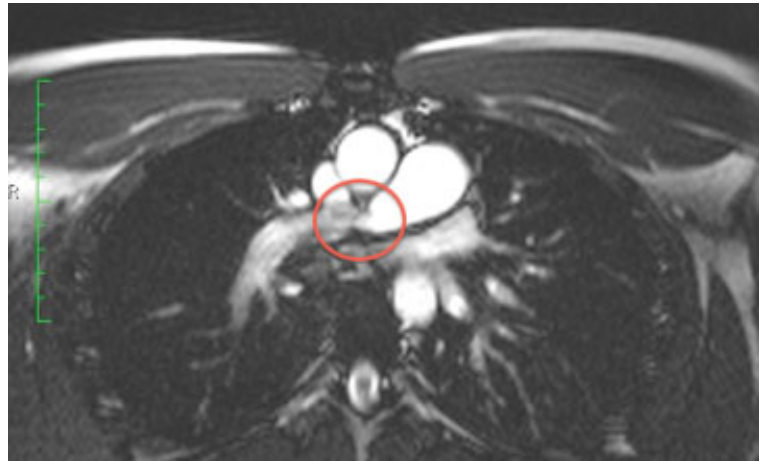

Fig. 1 Cardiac magnetic resonance imaging showing the central stenosis of the right pulmonary artery after the first operation.

artery pressure at exercise, and a pressure gradient between central and peripheral right pulmonary artery (-Table $\mathbf{1}$ ). Biplanar digital subtraction angiography showed bilateral pulmonary segmental artery stenoses and occlusions. Indication for surgery was based on the patient's individual loss of quality of life, pulmonary hemodynamics at exercise, and pulmonary artery obstructions at angiography.

Surgery was performed under extracorporeal circulation and two phases of deep hypothermic circulatory arrest (17 and 15 minutes). A complete endarterectomy of both pulmonary arteries was achieved and the central right pulmonary artery was reconstructed, using an autologous pericardial patch. The patient was extubated on postoperative day 1 and discharged from the hospital on the 14th day after an uneventful postoperative course. An oral anticoagulation with phenprocoumon was recommended for life time.

The patient was reevaluated after 3 months. He was in good clinical condition with normal physical capacity, corresponding to WHO functional class I. The right heart catheterization showed normal pulmonary hemodynamics at rest and at exercise. There was no pressure gradient between the central and the peripheral right pulmonary artery at rest, and $4 \mathrm{~mm} \mathrm{Hg}$ at exercise (-Table $\mathbf{1}$ ).

One year after the surgery, his physical capacity had further improved. MRI showed a normal dimension and function of the right ventricle (RVEF: 61\%). Pulmonary hemodynamics were normal at right heart catheterization
(-Table 1). Spiroergometry revealed a maximum work load of $200 \mathrm{~W}$ for 12 minutes with a maximum oxygen uptake of $45.9 \mathrm{~mL} / \mathrm{min} / \mathrm{kg}$.

\section{Discussion}

The actual case report describes a patient, who developed CTEPH in combination with a hemodynamically relevant stenosis of the central right pulmonary artery after thrombolysis and surgical embolectomy. The pathogenesis of the causal popliteal vein thrombosis of the patient could not be clarified and no coagulation abnormalities were found. Within the respective literature, you will find few references describing pulmonary embolism after prolonged running. The pulmonary artery stenosis was interpreted as a consequence of the first operation.

Assessing the presented case, the patient was treated according to current guidelines, which divide patients with pulmonary embolism into two major subgroups: high-risk and non-high-risk patients, describing the early mortality rate. Thrombolysis was unsuccessful probably due to the organization of the thrombotic material and recurrent episodes of acute embolism.

Interventional catheter-based fragmentation did not improve pulmonary hemodynamics. In literature, conclusive results of this procedure are still missing. Surgical embolectomy was then indicated. ${ }^{1-3}$

One to five percent of patients with acute pulmonary embolism develop CTEPH. ${ }^{4}$ Patients with persisting or recurrent dyspnea and acute pulmonary embolism in their medical history are screened with echocardiography and VQ scanning of the lungs. Right heart dysfunction and mismatched perfusion defects are suspicious of CTEPH. These patients should be transferred to an expert center to ensure the correct diagnosis by right heart catheterization and pulmonary angiography. An experienced interdisciplinary team should assess the operability. Using extracorporeal circulation and deep hypothermic circulatory arrest, pulmonary endarterectomy is the therapy of choice for patients with CTEPH, and it is a safe, standardized, and often curative treatment. ${ }^{5}$ Even patients in WHO functional class II should be evaluated, depending on their individual loss of quality of life, although they might not have CTEPH by definition (mean pressure in the pulmonary artery $\geq 25 \mathrm{~mm} \mathrm{Hg})^{4}$

Table 1 Right heart catheter before 3 months and 1 year after PEA

\begin{tabular}{|c|c|c|c|c|c|c|}
\hline \multirow[t]{2}{*}{ Right heart catheter } & \multicolumn{2}{|c|}{ Pre-op } & \multicolumn{2}{|c|}{ Post-op 3 mo } & \multicolumn{2}{|c|}{ Post-op $12 \mathrm{mo}$} \\
\hline & At rest & $150 \mathrm{~W}$ & At rest & $150 \mathrm{~W}$ & At rest & $125 \mathrm{~W}$ \\
\hline PAmean & 20 & 37 & 15 & 33 & 14 & 31 \\
\hline $\mathrm{Cl}$ & 3.4 & 6 & 3.45 & 11.2 & 3.2 & 8.8 \\
\hline PVR & 90 & 234 & 94 & 86.6 & 107 & 88.03 \\
\hline Gradient & 10 & 40 & 0 & 4 & - & - \\
\hline
\end{tabular}

Notes: All pressures were measured proximally to the stenosis. (PAmean = mean pressure in the pulmonary artery in mm $\mathrm{Hg}$; $\mathrm{Cl}=\mathrm{cardiac}$ index in $\mathrm{L} / \mathrm{min} / \mathrm{m}^{2}$; PVR = pulmonary vascular resistance in $\mathrm{dyn}^{*} \mathrm{sec} / \mathrm{cm}^{5}$; gradient $=$ pressure gradient central and peripheral of the stenosis of the right pulmonary artery in $\mathrm{mm} \mathrm{Hg}$ ). 
The patient was presented in WHO functional class II, but his individual loss of quality of life was distinct. Right-heart catheter showed borderline pulmonary hypertension at rest with pathological increase under work. Furthermore, there was a postoperative hemodynamically relevant stenosis of the right pulmonary artery after acute pulmonary embolectomy. The decision for surgery was made on individual basis regarding the explicit wish of the patient to have this surgery performed. A complete normalization of pulmonary hemodynamics and physical capacity on a long-term basis could be achieved by pulmonary endarterectomy with patch reconstruction of the right pulmonary artery.

\section{References}

1 Torbicki A, Perrier A, Konstantinides S, et al; ESC Committee for Practice Guidelines (CPG). Guidelines on the diagnosis and management of acute pulmonary embolism: the task force for the diagnosis and management of acute pulmonary embolism of the European Society of Cardiology (ESC). Eur Heart J 2008;29(18): 2276-2315

2 Hirsh J, Guyatt G, Albers GW, Harrington R, Schünemann HJ. Executive summary: American College of Chest Physicians Evidence-Based Clinical Practice Guidelines (8th Edition). Chest 2008;133(6, Suppl):71S-109S

3 Galiè N, Hoeper MM, Humbert M, et al; Task Force for Diagnosis and Treatment of Pulmonary Hypertension of European Society of Cardiology (ESC); European Respiratory Society (ERS); International Society of Heart and Lung Transplantation (ISHLT). Guidelines for the diagnosis and treatment of pulmonary hypertension. Eur Respir J 2009;34(6):1219-1263

4 Pepke-Zaba J, Delcroix M, Lang I, et al. Chronic thromboembolic pulmonary hypertension (CTEPH): results from an international prospective registry. Circulation 2011;124(18):1973-1981

5 Madani MM, Auger WR, Pretorius V, et al. Pulmonary endarterectomy: recent changes in a single institution's experience of more than 2,700 patients. Ann Thorac Surg 2012;94(1):97-103, discussion 103 